\title{
P3HT-graphene bilayer electrode for Schottky junction photodetectors
}

\author{
H Aydın ${ }^{1}$, S B Kalkan ${ }^{1}$, C Varlikli ${ }^{2}$ and C Çelebi ${ }^{1}$ (i) \\ ${ }^{1}$ Quantum Device Laboratory, Department of Physics, İzmir Institute of Technology, 35430, Izmir, Turkey \\ ${ }^{2}$ Department of Photonics, İzmir Institute of Technology, 35430, Izmir, Turkey \\ E-mail: cemcelebi@iyte.edu.tr
}

Received 10 December 2017, revised 18 January 2018

Accepted for publication 29 January 2018

Published 15 February 2018

\begin{abstract}
We have investigated the effect of a poly (3-hexylthiophene-2.5-diyl)(P3HT)-graphene bilayer electrode on the photoresponsivity characteristics of Si-based Schottky photodetectors. P3HT, which is known to be an electron donor and absorb light in the visible spectrum, was placed on CVD grown graphene by dip-coating method. The results of the UV-vis and Raman spectroscopy measurements have been evaluated to confirm the optical and electronic modification of graphene by the P3HT thin film. Current-voltage measurements of graphene $/ \mathrm{Si}$ and P3HT-graphene/Si revealed rectification behavior confirming a Schottky junction formation at the graphene/Si interface. Time-resolved photocurrent spectroscopy measurements showed the devices had excellent durability and a fast response speed. We found that the maximum spectral photoresponsivity of the P3HT-graphene/Si photodetector increased more than three orders of magnitude compared to that of the bare graphene/Si photodetector. The observed increment in the photoresponsivity of the P3HT-graphene/Si samples was attributed to the charge transfer doping from $\mathrm{P} 3 \mathrm{HT}$ to graphene within the spectral range between near-ultraviolet and near-infrared. Furthermore, the P3HT-graphene electrode was found to improve the specific detectivity and noise equivalent power of graphene/Si photodetectors. The obtained results showed that the P3HT-graphene bilayer electrodes significantly improved the photoresponsivity characteristics of our samples and thus can be used as a functional component in Si-based optoelectronic device applications.
\end{abstract}

Keywords: graphene, P3HT, electrode, photodetector

(Some figures may appear in colour only in the online journal)

\section{Introduction}

Graphene, as a one atom thick sheet of $\mathrm{sp}^{2}$ bonded carbon atoms arranged in a honeycomb lattice [1], forms a Schottky junction with rectification character when it is transferred onto most conventional semiconducting materials like $\mathrm{Si}$ [2], GaAs [3] and $\mathrm{SiC}$ [4]. In addition, owing to its high charge carrier mobility [5], superior optical transparency [6] and modulation of the Fermi level [7], graphene is considered as a transparent conductive electrode alternative to indium-thin-oxide (ITO) in microelectronic and optoelectronic devices operating with low leakage currents. Contrary to conventional metal/semiconductor photodetectors, where the Fermi level of the metal remains constant due to a high density of states, even small variations in the charge carrier density can significantly change the Fermi level of graphene, resulting in the modification of the Schottky barrier height and rectification behavior of the junction. Therefore, graphene/Si Schottky junction photodetectors have gained an increasing amount of attention over the past few years due to their photodetection capability at a broad wavelength range between $400-1100 \mathrm{~nm}$ [8-14]. However, the maximum value for the photoresponsivity of these devices is restricted to only about $0.3 \mathrm{~mA} \mathrm{~W}^{-1}$ as a consequence of their substantially low light absorption/gain ratio [8, 15]. For improving device photoresponsivity, the Fermi level of graphene should be lowered to increase both the magnitude of the built-in potential and junction electric field, which promote effective charge separation at the depletion region of the semiconducting material underneath $[8,16]$. Recently, a hybrid structure that 
combines graphene-Si quantum dots (Si-QDs) has been reported to enhance device photoresponsivity [16]. However, the photoresponsivity of this device is still limited to only about $0.5 \mathrm{~A} \mathrm{~W}^{-1}$ and besides it exhibits narrow band optical photodetection, greatly hindering the photodetector's wavelength detection capability especially between the nearultraviolet and near-infrared spectral range.

Recently, graphene-conductive polymer bilayer structures have attracted great attention since the conductive polymers provide a broad range of flexibility in their chemical and physical properties [17-19]. Among these conductive polymers, poly (3-hexylthiophene-2.5-diyl) $\mathrm{P} 3 \mathrm{HT}$ as an electron donor source is the most widely used material in optoelectronic devices [20-25], owing to its high absorption coefficient and electrical conductivity [26, 27]. Additionally, the (P3HT)-graphene bilayer structure shows effective charge transfer at the interface facilitating the movement of photogenerated holes from P3HT to graphene, whereas the photogenerated electrons still remain in $\mathrm{P} 3 \mathrm{HT}$ [28]. A recent study has shown that P3HT could be used to modify the Si surface as an electron blocking layer in a graphene/Si Schottky junction solar cell [29]. The power conversion efficiency of the device reaches to such a value that exceeds $10 \%$, suggesting a great potential for large scale photovoltaic applications. A P3HT-graphene hybrid composite has also been utilized as an active channel in photodetectors exhibiting great photocurrent and gain [28, 30].

In this work, we fabricated P3HT-graphene bilayer electrodes for use in Si-based Schottky junction photodetectors operating at a wide spectral wavelength range between 400-1100 nm. UV-vis absorbance and Raman spectroscopy measurements were conducted in order to determine the optical characteristics of the P3HT-coated graphene monolayer. The electronic and optoelectronic characterizations of the graphene/Si samples were done before and after coating the graphene electrodes with P3HT molecules. Time-resolved photocurrent spectroscopy measurements showed that the P3HT-graphene/Si samples exhibited enhanced photodetector performance compared to uncoated graphene/Si devices in terms of photoswitching characteristics, spectral responsivity, specific detectivity and noise equivalent power. The enhancement in the device performance is attributed to the effective charge transfer doping from P3HT to graphene under light illumination, which increases the magnitude of the built-in potential and widens the depletion region in the Si substrate underneath.

\section{Methods}

$\mathrm{Cu}$ foil (25 $\mu \mathrm{m}$ thick, 99.8 purity, Alfa Aesar) as the catalystsubstrate material was placed on a quartz plate and inserted into the tube furnace at atmospheric pressure in the chemical vapor deposition system. As for the first step, the $\mathrm{Cu}$ foil was heated up to $1073{ }^{\circ} \mathrm{C}$ under a $\mathrm{H}_{2}(20 \mathrm{sccm})+\operatorname{Ar}(1000 \mathrm{sccm})$ gas mixture with a temperature ramp rate of $30{ }^{\circ} \mathrm{C} \mathrm{min}^{-1}$. Then the foil was annealed under the same temperature and flow rates for $1 \mathrm{~h}$. After the annealing process, $\mathrm{CH}_{4}(10 \mathrm{sccm})$ was introduced into the tube furnace for $1 \mathrm{~min}$ in order to facilitate the graphene growth. Finally, the sample was left for rapid cooling from growth temperature to room temperature under gas flows of $\mathrm{H}_{2}(20 \mathrm{sccm})$ and $\mathrm{Ar}(1000 \mathrm{sccm})$.

Microposit S1318 photoresist (PR) was utilized as the supporting layer during the graphene transfer process. The PR on the graphene- $\mathrm{Cu}$ template was drop casted and annealed at $70{ }^{\circ} \mathrm{C}$ overnight. Iron chloride $\left(\mathrm{FeCl}_{3}\right)$ solution was used to etch the $\mathrm{Cu}$ foil and to suspend the graphene-PR. After the $\mathrm{Cu}$ foil was fully etched away, graphene-PR was rinsed with deionized water in order to remove any $\mathrm{FeCl}_{3}$ residue. After the $\mathrm{N}_{2}$ drying process, graphene-PR was transferred onto the surface of the clean $\mathrm{SiO}_{2} / \mathrm{n}-\mathrm{Si}$ substrate. The substrate was baked at a temperature of $110{ }^{\circ} \mathrm{C}$ in order to provide better adhesion of the graphene layer to the surface of the substrate. As for the last step, the PR was removed by hot acetone to leave the graphene layer on its own on the $\mathrm{SiO}_{2} / \mathrm{n}-\mathrm{Si}$ substrate.

For the experiments, we used commercial $\mathrm{SiO}_{2}$ $(300 \mathrm{~nm}) / \mathrm{n}-\mathrm{Si}$ wafers with a resistivity of $\rho=1-10 \Omega . \mathrm{cm}$. The wafer was diced into $10 \mathrm{~mm} \times 10 \mathrm{~mm}$ substrates and was ultrasonically cleaned for $10 \mathrm{~min}$ in deionized water, acetone, ethanol and 2-proponal, respectively. Thereafter, a part of the $\mathrm{SiO}_{2}$ using a mixture of $\mathrm{H}_{2} \mathrm{O}: \mathrm{HNO}_{3}: \mathrm{HF}(60: 1: 1.5)$ was etched in order to prevent electrical shortening along the graphene layer. Then, the graphene was transferred onto the surface of the partially etched $\mathrm{SiO}_{2} / \mathrm{n}-\mathrm{Si}$ substrate. The interconnecting electrodes comprising of $5 \mathrm{~nm} \mathrm{Cr}$ and $85 \mathrm{~nm}$ $\mathrm{Au}$ layers were deposited on the $\mathrm{SiO}_{2}$ and $\mathrm{n}-\mathrm{Si}$ to get ohmic contact using the thermal evaporation technique. A set of regioregular P3HT solutions with $2.5,5$ and $10 \mathrm{mg} \mathrm{ml}^{-1}$ concentrations were prepared at room temperature in toluene. Then, the coating of the P3HT molecules was completed using the dip-coating technique and extracted at a constant speed of $5 \mathrm{~mm} \mathrm{~s}^{-1}$. Finally, the P3HT-graphene/Si samples were dried at $110^{\circ} \mathrm{C}$ for $10 \mathrm{~min}$ in air.

The UV-vis absorption spectrum of the P3HT-graphene on the quartz substrate was measured for a wavelength range of 400-990 nm. Raman spectrum of the graphene transferred onto the $\mathrm{SiO}_{2} / \mathrm{n}-\mathrm{Si}$ was acquired using a $532 \mathrm{~nm}$ laser excitation with 600 groove $\mathrm{mm}^{-1}$ grating under $100 \mathrm{X}$ microscope objective (XploRA Horiba). In order to perform optoelectronic characterization of the graphene/Si photodetector and P3HT-graphene/Si, a tungsten-halogen lamp (Osram, $275 \mathrm{~W}$ ) was used to generate light and specific wavelengths were separated with the help of a monochromator (Newport, Oriel Cornerstone), which included an internal shutter. A spectrophotometer (Oceans Optics) was used to calibrate the full width of half maximum of light by changing the slit of the spectrophotometer and the power output of the commercial $\mathrm{Si}$ photodetector (FDS10X10, Thorlabs) was obtained to define the incoming power of light on the device area. Then timeresolved dark current and photocurrent, and responsivity versus wavelength (resolution $15 \mathrm{~nm}$ ) measurements were employed by using a Keithley 2400 source-meter and Keithley 6485 picoammeter. 

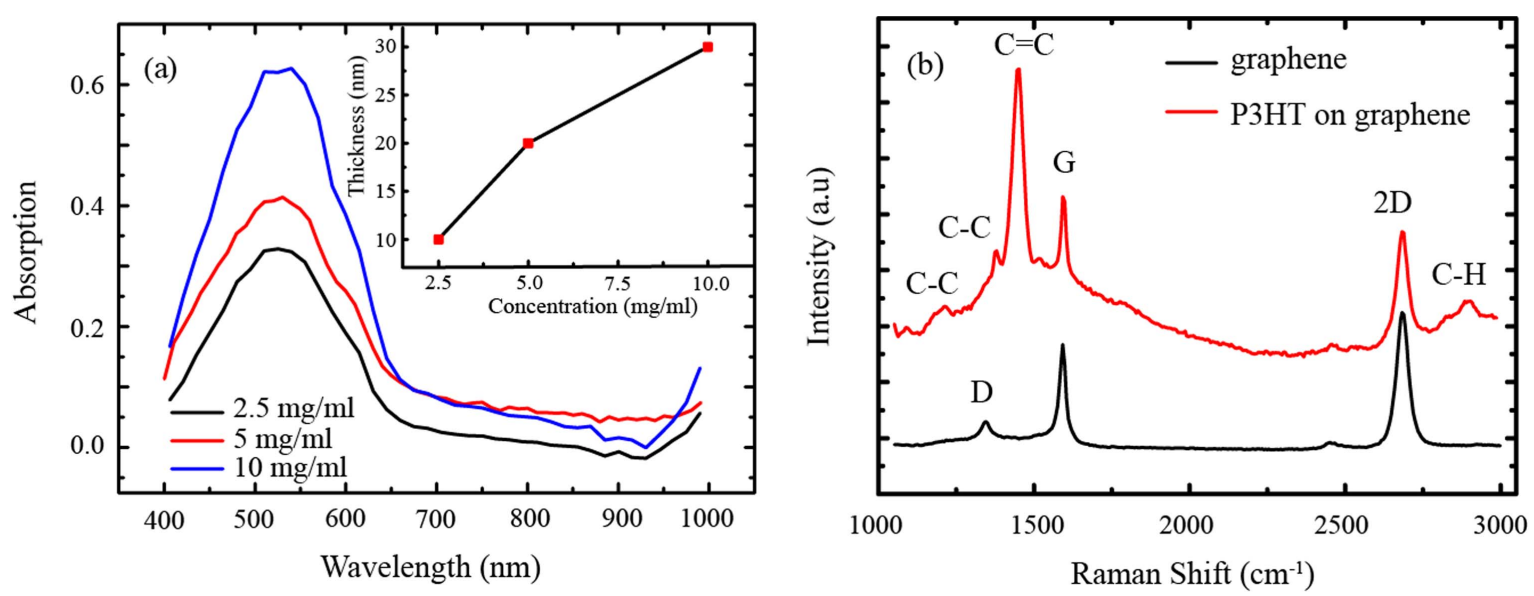

Figure 1. (a) Optical absorption spectra of P3HT films prepared from different concentrations on quartz. Inset: thickness of P3HT as a function of concentration. (b) Raman spectra of graphene and P3HT on graphene transferred onto $\mathrm{SiO}_{2}$ coated $\mathrm{Si}$.

(a)

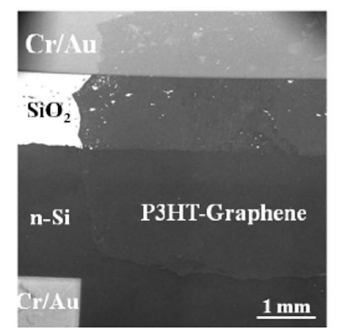

(b)

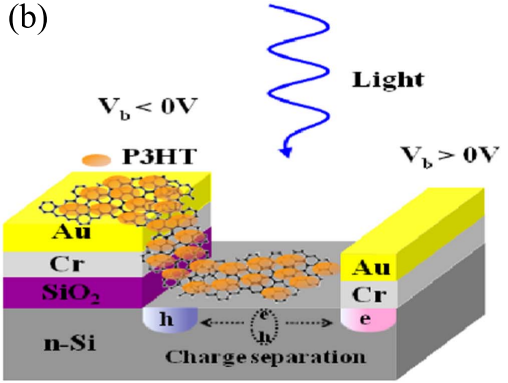

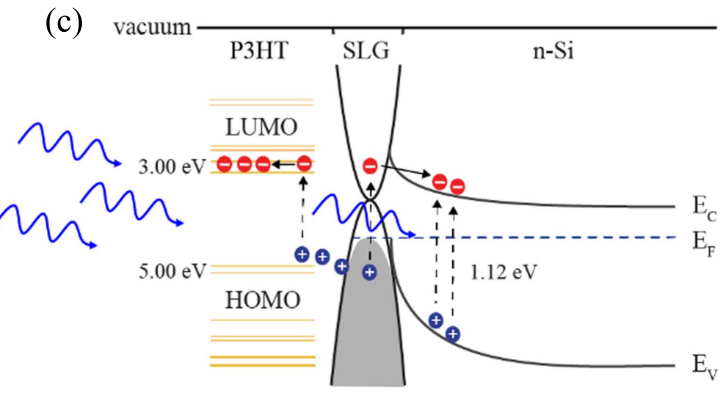

Figure 2. (a) Low-magnification SEM image of the fabricated P3HT-graphene/Si device. (b) Schematic cross-section view of the biased P3HT-graphene/Si device. The dashed arrow describes separation of photogenerated charge carriers. (c) Energy band diagram of P3HTgraphene/Si photodetectors.

\section{Results and discussion}

Figure 1(a) shows the optical spectrum of the P3HT-graphene film on quartz substrate. The P3HT film exhibits a main absorption peak at a wavelength of around $535 \mathrm{~nm}$. This is attributed to the $\pi-\pi^{*}$ electronic transition and lattice vibration in crystalline P3HT domains. Concentration dependent thickness variations of dip-coated P3HT films are presented in the inset of figure 1(a). The measured thicknesses of the P3HT films were about 10, 20 and $30 \mathrm{~nm}$ corresponding to solution concentrations of $2.5,5$ and $10 \mathrm{mg} \mathrm{ml}^{-1}$, respectively.

Figure 1(b) shows the Raman spectra of graphene and P3HT film on graphene. In general, the Raman spectrum of graphene exhibits typical band-peaks at the wavenumbers of D $\left(1345 \mathrm{~cm}^{-1}\right), \mathrm{G}\left(1593 \mathrm{~cm}^{-1}\right)$ and $2 \mathrm{D}\left(2685 \mathrm{~cm}^{-1}\right)$. The $\mathrm{G}$ band is the first Raman peak related to $\mathrm{C}-\mathrm{C}$ stretching of $\mathrm{sp}^{2}$ carbon. The 2D band is the second order graphene peak and the $\mathrm{D}$ band provides information on the amount of disorder in graphene. The intensity ratios of $G / 2 D$ and $D / G$ were used to determine the number of graphene layers and defect content of graphene. In our Raman analysis, $\mathrm{I}_{\mathrm{G} / 2 \mathrm{D}}$ and $\mathrm{I}_{\mathrm{D} / \mathrm{G}}$ are about 0.6 and 0.1 , respectively, confirming the presence of monolayer graphene [31]. P3HT film has different modes; $1452 \mathrm{~cm}^{-1}$ for $\mathrm{C}=\mathrm{C}$ skeleton symmetric stretching, $1379 \mathrm{~cm}^{-1}$ for $\mathrm{C}-\mathrm{C}$ skeletal stretching, $2896 \mathrm{~cm}^{-1}$ for $\mathrm{C}-\mathrm{H}$ stretching and $1208 \mathrm{~cm}^{-1}$ for inter-ring $\mathrm{C}-\mathrm{C}$ stretch mode [32]. The main Raman peak of the P3HT-coated graphene film at $1596 \mathrm{~cm}^{-1}$ and 2D peak at $2785 \mathrm{~cm}^{-1}$ are also present (figure 1(b)). However, the D peak is suppressed by $\mathrm{C}-\mathrm{C}$ stretching of $\mathrm{P} 3 \mathrm{HT}$ and the $\mathrm{G}$ peak is shifted from $1593-1596 \mathrm{~cm}^{-1}$. This manifests that charge transfer occurs between P3HT and graphene [32].

Figure 2(a) shows a scanning electron microscopy (SEM) image of the $\mathrm{P} 3 \mathrm{HT}$-graphene/Si photodetector retaining P3HT-graphene film and $\mathrm{Cr} / \mathrm{Au}$ electrode both on the $\mathrm{n}-\mathrm{Si}$ and $\mathrm{SiO}_{2}$ sides of the device structure. Figure 2(b) depicts the three-dimensional view of the biased $\mathrm{P} 3 \mathrm{HT}$-graphene/Si device. The device with P3HT-graphene of a P3HT-graphene electrode and an underlying n-type $\mathrm{Si}$ substrate, which was in contact with $3 \mathrm{~mm} \times 5 \mathrm{~mm}$ of the device area. $\mathrm{Cr} / \mathrm{Au}$ was used to get ohmic contacts both on the graphene and on the $\mathrm{n}-\mathrm{Si}$. Upon light illumination in the visible wavelength range, electron-hole pairs were generated in the P3HT layer and in the depletion region of the n-type Si substrate as well. When a bias voltage was applied between the two electrodes, the electric field in the depletion region sweeped the photogenerated electrons through to the n-type $\mathrm{Si}$ which was forward biased $\left(V_{b}<0 \mathrm{~V}\right)$, whereas the holes moved towards the region underneath, which was reverse biased $\left(V_{b}>0 \mathrm{~V}\right)$ $\mathrm{P} 3 \mathrm{HT} /$ graphene. Because of the fact that the photogenerated charge carriers were collected separately at the corresponding 

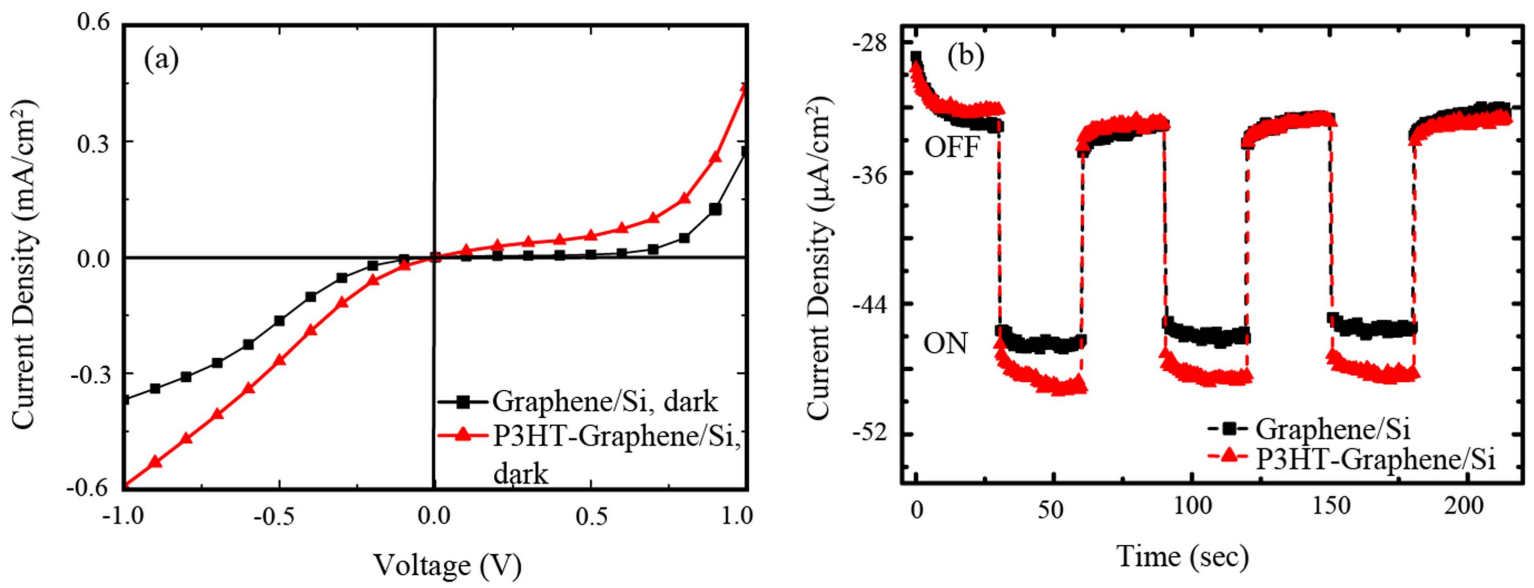

Figure 3. (a) The $J-V$ curve of the graphene/Si and P3HT-graphene/Si in dark. (b) Photoswitching behavior of the graphene/Si and P3HTgraphene/Si photodetectors under $540 \mathrm{~nm}$ light with an intensity of $20 \mu \mathrm{W}$ at bias voltage of $-0.1 \mathrm{~V}$.

Table 1. Summary of the performances of the graphene based Si photodetectors.

\begin{tabular}{lccccc}
\hline Device Structure & $I_{\text {dark }}(\mathrm{nA})$ & $R\left(\mathrm{~A} \mathrm{~W}^{-1}\right)$ & $\mathrm{D}^{*}\left(10^{9}\right)$ (Jones) & $\mathrm{NEP}\left(\mathrm{pW} / \mathrm{Hz}^{-1 / 2}\right)$ & Reference \\
\hline Graphene/Si & 6 & 0.24 & 19 & 0.20 & This work \\
P3HT-graphene/Si & 40 & 0.78 & 26 & 0.14 & This work \\
Graphene/Si & - & 0.43 & 7.7 & 1.0 & {$[8]$} \\
Graphene/Si & - & 0.23 & - & - & {$[13]$} \\
Ag-Nps/graphene/Si & - & 0.10 & - & - & {$[10]$} \\
Graphene/Si-QDs/Si & - & 0.35 & 1.0 & 6.7 & {$[15]$} \\
Si-QDs/graphene/Si & - & 0.50 & 7.4 & &
\end{tabular}

electrodes, this yielded a measurable photocurrent in the device. Figure 2(c) demonstrates the schematic illustration for the energy-band diagram of the P3HT-graphene/Si photodetector under reverse bias conditions. When the P3HTgraphene and $\mathrm{n}-\mathrm{Si}$ came into contact, a depletion region and Schottky barrier $\left(\varphi_{b}\right)$ were formed at the graphene $/ \mathrm{n}-\mathrm{Si}$ interface due to the difference between their corresponding Fermi levels [2, 33, 34]. Hence, the electrons in n-Si tended to flow into the graphene until the Fermi levels were aligned across the junction. Under illumination, the incident light went through the P3HT-graphene bilayer electrode and penetrated into the $\mathrm{n}-\mathrm{Si}$ to generate electron-hole pairs. The holes formed in the P3HT layer were transferred to the graphene via charge transfer doping, leading to p-type doping which increases built-in potential and junction field [16, 28]. This gave rise to the efficient separation of the photogenerated charge carriers in the depletion region and the holes moved to the graphene, whereas the electrons were transferred to the $\mathrm{Si}$, enhancing the photocurrent of the device.

As will be discussed further, the device with $20 \mathrm{~nm}$ thick P3HT film displays the best performance in terms of current density $(J)$-voltage $(V)$ characteristics and time-dependent photocurrent behavior, this is presented in figures 3(a) and (b). Both the graphene/Si and P3HT-graphene/Si photodetectors exhibited rectification behavior confirming the Schottky junction formation at the graphene/Si interface. Additionally, while the dark current of the graphene/Si device at zero bias was about $6 \mathrm{nA}$, the P3HT-based device was measured as $40 \mathrm{nA}$ (table 1). Such a small increment of dark current is attributed to the presence of surface states with high density. Time-dependent photocurrent measurements of graphene/Si and $\mathrm{P} 3 \mathrm{HT}$-graphene/Si photodetectors were conducted over several switching on/off cycles under $540 \mathrm{~nm}$ light illumination at a bias voltage of $-0.1 \mathrm{~V}$. The measurements were done during a total time of $210 \mathrm{~s}$ at $30 \mathrm{~s}$ intervals. As shown in figure 3(b), the measured current of the devices displays two different states at relatively low incident light power $(20 \mu \mathrm{W})$; a low-current state at around $32 \mu \mathrm{A}$ in dark and average photocurrents of about 46 and $50 \mu \mathrm{A}$ for the graphene/Si and P3HT-graphene/Si photodetectors, respectively. Additionally, for the $\mathrm{P} 3 \mathrm{HT}$-graphene/Si photodetector, upon light irradiation, a sharp increase in the current was followed by an exponential decay. As the light was turned off, a rapid downward trend in the current can be seen, following an exponential growth. The time constants for the growth and decay of the photocurrents were determined as 4.7 and $8.1 \mathrm{~s}$, respectively. Furthermore, photoswitching characteristics of both devices show a reversible photocurrent behavior and good stability. Our device with P3HT molecules was stable against air-exposure up to a period of about five days. However, after three months, the device was affected due to the adsorption of atmospheric molecules on the P3HT film, resulting in a degradation of device performance and stability $[35,36]$. Therefore, the device needs to be passivated with a proper encapsulation layer for long-term device stability. 

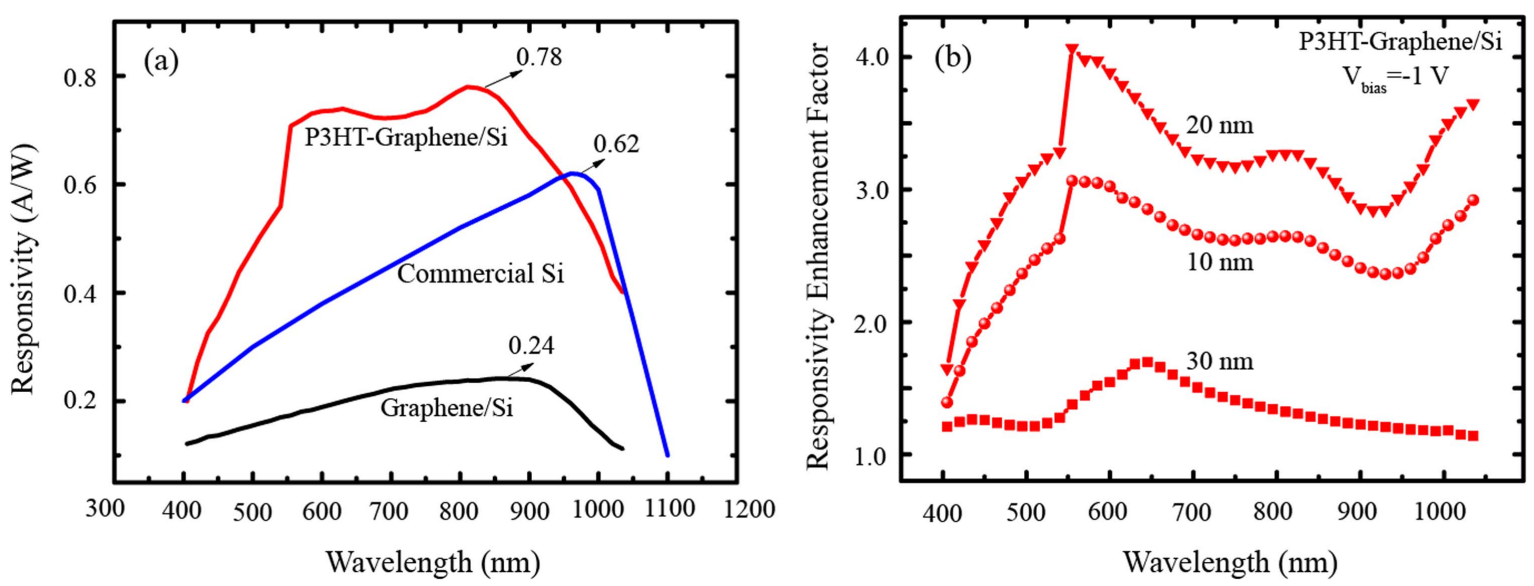

Figure 4. (a) Responsivity of graphene/Si, P3HT-graphene/Si and commercial Si photodetectors under bias of $-1 \mathrm{~V}$. (b) Responsivity enhancement factor of P3HT-based devices at different thicknesses.

Photoresponsivity is one of the most important parameters for the light sensing capability of photodetector devices and can be written as [37]

$$
R=\frac{I_{\text {photo }}-I_{\mathrm{dark}}}{P}
$$

where $I_{\text {photo }}$ is the photocurrent, $I_{\text {dark }}$ is the dark current and $P$ is the optical power of incident light. Figure 4(a) displays spectral responsivity of the graphene/Si, P3HT-graphene/Si and commercial (Thorlabs) $\mathrm{Si}$ photodetectors under an applied reverse bias of $1 \mathrm{~V}$. The graphene/Si photodetector exhibited typical Si-based responsivity behavior similar to that reported in previous literature $[13,16]$. The obtained maximum responsivity reached $0.24 \mathrm{~A} \mathrm{~W}^{-1}$ at a wavelength of $850 \mathrm{~nm}$, in correlation with typical optical absorption spectrum of n-type Si. However, the respective photoresponsivity exhibited a downward trend below the cutoff wavelength of $1100 \mathrm{~nm}$ that corresponded to an energy level, which was below the band gap energy of Si. For the commercial photodetector, the photoresponsivity with a linear character was present up to a wavelength of $950 \mathrm{~nm}$ and then decayed due to the Si absorption edge.

The P3HT-graphene bilayer electrode based Si photodetector presents not only higher responsivity but also wider wavelength coverage with respect to graphene/Si and commercial Si photodetectors. The obtained maximum responsivity at a wavelength of $850 \mathrm{~nm}$ is about $0.78 \mathrm{~A} \mathrm{~W}^{-1}$. It is clear that the maximum responsivity of our P3HT-graphene/ Si device was more than three orders and one order of magnitude higher than the maximum responsivities of the graphene/Si and commercial Si photodetectors, respectively. The responsivity started to increase at the absorption onset wavelength of P3HT (figure 1(a)) and reached a maximum value around $850 \mathrm{~nm}$, benefiting from the $\mathrm{Si}$ absorption. However, the slope of the decay observed for the wavelengths above $990 \mathrm{~nm}$ was much lower than that of the one observed in the commercial Si photodetector. This can be attributed to the P3HT-induced increment of responsivity at the higher wavelengths. Upon light illumination, the holes in P3HT were transferred to the graphene layer, which is unintentionally p-doped [38, 39], resulting in lowering the Fermi level of graphene which led to a larger built-in potential between $n-S i$ and graphene. A larger built-in potential gives rise to a wider depletion region providing efficient dissociation of photongenerated carriers $[16,40]$. A similar manner of the responsivity curve at a higher wavelength $(>990 \mathrm{~nm})$ can be seen in the literature [16]. Furthermore, the photodetection seen at the wavelengths $>990 \mathrm{~nm}$ can also be related to the localized polaron absorption of P3HT exceeding up to $1100 \mathrm{~nm}$ [41]. As displayed in figure 4(b), changing the thickness of P3HT caused an alteration in the responsivity enhancement factors. When the thickness of P3HT was increased from 10-20 nm, the responsivity enhancement factor also increased and displayed two distinct peaks at around 540 and $850 \mathrm{~nm}$ originating from the absorption of $\mathrm{P} 3 \mathrm{HT}$ and $\mathrm{Si}$, respectively. However, the $30 \mathrm{~nm}$ thick P3HT film exhibited a red-shift due to the agglomeration of $\mathrm{P} 3 \mathrm{HT}$ and decreased the responsivity enhancement factor. Increasing the thickness of P3HT may increase the density of electrons in the excited states upon irradiation and consequently facilitate the hole doping of graphene. However, when the exciton diffusion length of regioregular P3HT is considered [42], in such a thick film, the photogenerated excitons may also recombine before reaching the P3HT-graphene interface and cause a decrease in the responsivity enhancement factor.

The specific detectivity $\left(D^{*}\right)$ is one of figures of merit for a photodetector and can be given as [14]

$$
D^{*}=\frac{A^{1 / 2} R}{\sqrt{2 e I_{\mathrm{d}}}}
$$

where $A$ is the active area of the photodetector, $R$ is the responsivity, $e$ is the elementary charge and $I_{\mathrm{d}}$ is the dark current. As shown in figure 5(a), the calculated $D^{*}$ of graphene/Si device is about $19 \times 10^{9}$ Jones, whereas the $D^{*}$ of the P3HT-graphene/Si photodetector exceeded $26 \times 10^{9}$ Jones at $850 \mathrm{~nm}$, which is higher than the values reported in similar work [8, 16]. Apparently, the P3HT-graphene bilayer electrode improved the detection limit of the graphene/Si photodetector. There is reversal $\mathrm{D}^{*}$ between the graphene/ $\mathrm{Si}$ 

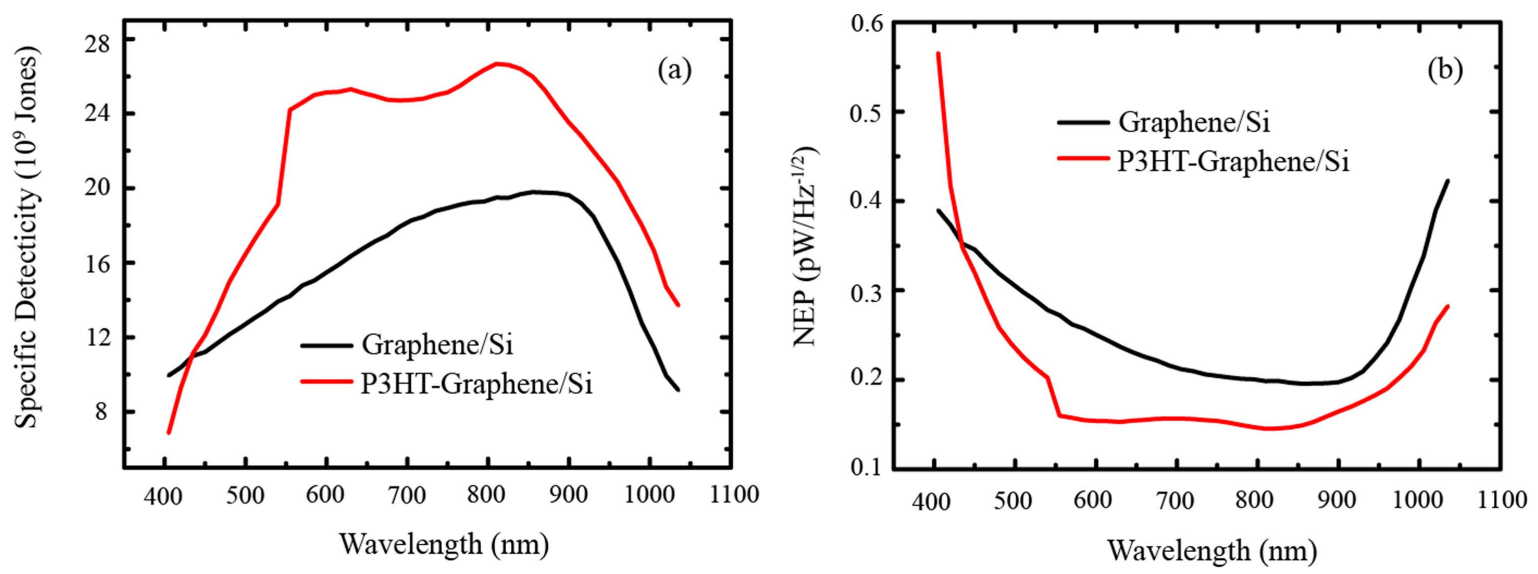

Figure 5. (a) Spectral dependence of $\mathrm{D}^{*}$ and (b) NEP of the graphene/Si and P3HT-graphene/Si photodetectors.

and P3HT-graphene/Si photodetectors at approximately $450 \mathrm{~nm}$. Considering the dark currents of both devices, the responsivity of the P3HT-graphene/Si photodetector was not high enough compared to the graphene/Si photodetector at lower wavelengths due to the absorption of the P3HT and Si. This is because of the fact that the $\mathrm{D}^{*}$ of the graphene/Si device increased with respect to the $\mathrm{D}^{*}$ of the P3HT modified graphene/Si photodetector at wavelengths below $450 \mathrm{~nm}$.

Noise equivalent power (NEP) describes the incident power required to obtain a signal to noise ratio of 1 at a bandwidth of $1 \mathrm{~Hz}$. The NEP can be obtained by using the Flicker noise $(1 / \mathrm{f})$, shot noise and thermal noise of a device and this gives information about the minimum detectable signal of the photodetector [37, 43-45] and can be written as

$$
\mathrm{NEP}=\frac{A^{1 / 2}}{D^{*}}
$$

Figure 5(b) shows the spectral NEP of the graphene/Si and $\mathrm{P} 3 \mathrm{HT}$-graphene/Si photodetectors. The obtained minimum $\mathrm{NEP}$ is about $0.20 \mathrm{pW} \mathrm{Hz}^{-1 / 2}$ for graphene $/ \mathrm{Si}$ at $850 \mathrm{~nm}$. However, the device with the P3HT-graphene bilayer electrode exhibited low NEP down to $0.14 \mathrm{pW} \mathrm{Hz}^{-1 / 2}$ at a wavelength of $850 \mathrm{~nm}$. This value is higher than that reported in a similar work [8]. Additionally, the NEP values of the P3HT-graphene/Si photodetector changed from $0.15-0.14 \mathrm{pW} \mathrm{Hz}^{-1 / 2}$ for a wavelength range between $550-885 \mathrm{~nm}$. Such a small variation in the NEP value enables the device to operate at a broader bandwidth ranging from the visible to the near-infrared spectrum.

The performance characteristics of the Si photodetectors with graphene electrodes are compared in table 1 with respect to their reference samples. It is clear that the P3HT-graphene bilayer electrode improved the performance of the respective photodetector in terms of the device parameters of $R, D^{*}$ and NEP. As compared to previously reported graphene-based $\mathrm{Si}$ photodetectors, the photoresponsivity of the graphene/Si device was restricted to about $0.40 \mathrm{~A} \mathrm{~W}^{-1}$ due to low light absorption/ gain ratio. Additionally, plasmonic silver nanoparticles (Ag-Nps) on the graphene electrode were used to enhance the light interaction and provide an efficient Schottky junction formation [10]. However, the use of these $\mathrm{Ag}-\mathrm{Nps}$ hindered and limited the photoresponsivity of the device at around $0.1 \mathrm{~A} \mathrm{~W}^{-1}$. Furthermore, the Si-QD-graphene coupled electrode was used to increase the built-in potential. The device was shown to exhibit relatively high $R, D^{*}$ and NEP values [16].

\section{Conclusion}

In this work, the optoelectronic characteristics of graphene/ $\mathrm{Si}$ and P3HT-graphene/Si Schottky photodetectors were studied. The obtained results reveal that: (i) P3HT-graphene can be used as a bilayer electrode for the graphene/Si photodetector; (ii) UV-vis and Raman spectroscopy verified the modification of graphene by the $\mathrm{P} 3 \mathrm{HT}$ thin film; (iii) the $J-V$ characteristics of the bare and P3HT-coated photodetectors indicate rectification behavior, confirming Schottky junction formation at the graphene-Si interface; (iv) the photoswitching characteristic of both devices displays reversible behavior, affirming good stability; (v) P3HT-graphene/Si shows better photodetector performance than bare devices in terms of photoswitching characteristics, spectral responsivity, specific detectivity and noise equivalent power; (vi) enhancing the photoresponsivity of the P3HT-graphene/Si device is directly related to Fermi level tuning of graphene via charge transfer doping from P3HT to graphene. The experimental results clearly show that the robust characteristics, structural stability and higher photoresponse of the P3HT-graphenebased bilayer electrode holds promise for Si-based optoelectronic devices.

\section{Acknowledgments}

The authors would like to thank Volga Bulmuş for Raman measurements and Sparks Electronics Ltd Turkey for providing us with the stainless steel hard masks that were used in the device fabrication processes. 


\section{ORCID iDs}

\section{C Çelebi (ib https://orcid.org/0000-0003-1070-1129}

\section{References}

[1] Novoselov K S, Geim A K, Morozov S, Jiang D, Katsnelson M, Grigorieva I, Dubonos S and Firsov A A 2005 Two-dimensional gas of massless Dirac fermions in graphene Nature 438 197-200

[2] Kalita G, Hirano R, Ayhan M E and Tanemura M 2013 Fabrication of a Schottky junction diode with direct growth graphene on silicon by a solid phase reaction J. Phys. D: Appl. Phys. 46455103

[3] Tomer D, Rajput S, Hudy L, Li C and Li L 2015 Inhomogeneity in barrier height at graphene/Si (GaAs) Schottky junctions Nanotechnology 26215702

[4] Shivaraman S, Herman L H, Rana F, Park J and Spencer M G 2012 Schottky barrier inhomogeneities at the interface of few layer epitaxial graphene and silicon carbide Appl. Phys. Lett. 100183112

[5] Bolotin K I, Sikes K, Jiang Z, Klima M, Fudenberg G, Hone J, Kim P and Stormer H 2008 Ultrahigh electron mobility in suspended graphene Solid State Commun. 146 351-5

[6] Nair R R, Blake P, Grigorenko A N, Novoselov K S, Booth T J, Stauber T, Peres N M and Geim A K 2008 Fine structure constant defines visual transparency of graphene Science 3201308

[7] Yu Y-J, Zhao Y, Ryu S, Brus L E, Kim K S and Kim P 2009 Tuning the graphene work function by electric field effect Nano Lett. $93430-4$

[8] An X, Liu F, Jung Y J and Kar S 2013 Tunable graphenesilicon heterojunctions for ultrasensitive photodetection Nano Lett. 13 909-16

[9] Lv P, Zhang X, Zhang X, Deng W and Jie J 2013 Highsensitivity and fast-response graphene/crystalline silicon schottky junction-based near-IR photodetectors IEEE Electron Device Lett. 34 1337-9

[10] Ayhan M E, Kalita G, Kondo M and Tanemura M 2014 Photoresponsivity of silver nanoparticles decorated graphene-silicon Schottky junction RSC Adv. 4 26866-71

[11] Zhu M, Li X, Guo Y, Li X, Sun P, Zang X, Wang K, Zhong M, Wu D and Zhu H 2014 Vertical junction photodetectors based on reduced graphene oxide/silicon Schottky diodes Nanoscale 6 4909-14

[12] Chen Z, Cheng Z, Wang J, Wan X, Shu C, Tsang H K, Ho H P and Xu J B 2015 High responsivity, broadband, and fast graphene/silicon photodetector in photoconductor mode Adv. Opt. Mater. 3 1207-14

[13] Riazimehr S, Bablich A, Schneider D, Kataria S, Passi V, Yim C, Duesberg G S and Lemme M C 2016 Spectral sensitivity of graphene/silicon heterojunction photodetectors Solid-State Electron. 115 207-12

[14] Wan X, Xu Y, Guo H, Shehzad K, Ali A, Liu Y, Yang J, Dai D, Lin C-T and Liu L 2017 A self-powered highperformance graphene/silicon ultraviolet photodetector with ultra-shallow junction: breaking the limit of silicon? npj $2 D$ Mater. Appl. 14

[15] Shin D H, Kim S, Kim J M, Jang C W, Kim J H, Lee K W, Kim J, Oh S D, Lee D H and Kang S S 2015 Graphene/Siquantum-dot heterojunction diodes showing high photosensitivity compatible with quantum confinement effect Adv. Mater. 27 2614-20

[16] Yu T, Wang F, Xu Y, Ma L, Pi X and Yang D 2016 Graphene coupled with silicon quantum dots for high-performance bulk-silicon-based Schottky-junction photodetectors $A d v$. Mater. 28 4912-9

[17] Li Y, Guo Q, Li Z, Pei J and Tian W 2010 Solution processable D-A small molecules for bulk-heterojunction solar cells Energy Environ. Sci. 3 1427-36

[18] Liu Z, Liu Q, Huang Y, Ma Y, Yin S, Zhang X, Sun W and Chen Y 2008 Organic photovoltaic devices based on a novel acceptor material: graphene Adv. Mater. 20 3924-30

[19] Baeg K J, Binda M, Natali D, Caironi M and Noh Y Y 2013 Organic light detectors: photodiodes and phototransistors Adv. Mater. 25 4267-95

[20] Zhang L, Li Y, Shi J, Shi G and Cao S 2013 Nonvolatile rewritable memory device based on solution-processable graphene/poly (3-hexylthiophene) nanocomposite Mater. Chem. Phys. 142 626-32

[21] Reyes-Reyes M, Kim K, Dewald J, López-Sandoval R, Avadhanula A, Curran S and Carroll D L 2005 Mesostructure formation for enhanced organic photovoltaic cells Org. Lett. 7 5749-52

[22] Lai Y C, Wang Y X, Huang Y C, Lin T Y, Hsieh Y P, Yang Y J and Chen Y F 2014 Rewritable, moldable, and flexible sticker-type organic memory on arbitrary substrates Adv. Funct. Mater. 24 1430-8

[23] Berson S, De Bettignies R, Bailly S and Guillerez S 2007 Poly (3-hexylthiophene) fibers for photovoltaic applications $A d v$. Funct. Mater. 17 1377-84

[24] Wu Y, Zhang X, Jie J, Xie C, Zhang X, Sun B, Wang Y and Gao P 2013 Graphene transparent conductive electrodes for highly efficient silicon nanostructures-based hybrid heterojunction solar cells J. Phys. Chem. C 117 11968-76

[25] Huang J, Hines D R, Jung B J, Bronsgeest M S, Tunnell A, Ballarotto V, Katz H E, Fuhrer M S, Williams E D and Cumings J 2011 Polymeric semiconductor/graphene hybrid field-effect transistors Org. Electron. 12 1471-6

[26] Saini V, Li Z, Bourdo S, Dervishi E, Xu Y, Ma X, Kunets V P, Salamo G J, Viswanathan T and Biris A R 2009 Electrical, optical, and morphological properties of P3HT-MWNT nanocomposites prepared by in situ polymerization $J$. Phys. Chem. C 113 8023-9

[27] Miller S, Fanchini G, Lin Y-Y, Li C, Chen C-W, Su W-F and Chhowalla M 2008 Investigation of nanoscale morphological changes in organic photovoltaics during solvent vapor annealing J. Mater. Chem. 18 306-12

[28] Tan W C, Shih W H and Chen Y F 2014 A highly sensitive graphene-organic hybrid photodetector with a piezoelectric substrate Adv. Funct. Mater. 24 6818-25

[29] Xie C, Zhang X, Wu Y, Zhang X, Zhang X, Wang Y, Zhang W, Gao P, Han Y and Jie J 2013 Surface passivation and band engineering: a way toward high efficiency graphene-planar Si solar cells J. Mater. Chem. A 1 8567-74

[30] Huisman E H, Shulga A G, Zomer P J, Tombros N, Bartesaghi D, Bisri S Z, Loi M A, Koster L J A and van Wees B J 2015 High gain hybrid graphene-organic semiconductor phototransistors ACS Appl. Mater. Interfaces 7 11083-8

[31] Lin Z, Ye X, Han J, Chen Q, Fan P, Zhang H, Xie D, Zhu H and Zhong M 2015 Precise control of the number of layers of graphene by picosecond laser thinning Sci. Rep. 511662

[32] Bkakri R, Kusmartseva O E, Kusmartsev F, Song M and Bouazizi A 2015 Degree of phase separation effects on the charge transfer properties of P3HT: graphene nanocomposites J. Lumin. 161 264-70

[33] Kumar R, Varandani D and Mehta B 2016 Nanoscale interface formation and charge transfer in graphene/silicon Schottky junctions; KPFM and CAFM studies Carbon 98 41-9

[34] Liu J, Yin Y, Yu L, Shi Y, Liang D and Dai D 2017 Silicongraphene conductive photodetector with ultra-high responsivity Sci. Rep. 740904 
[35] Kapoor A K, Kumar U, Balakrishnan V and Basu P 2007 Degradation process in organic thin film devices fabricated using P3HT Pramana 68 489-98

[36] Zhao Y, Sugunan A, Schmidt T, Fornara A, Toprak M S and Muhammed M 2014 Relaxation is the key to longer life: suppressed degradation of P3HT films on conductive substrates J. Mater. Chem. A 2 13270-6

[37] Li J, Niu L, Zheng Z and Yan F 2014 Photosensitive graphene transistors Adv. Mater. 26 5239-73

[38] Suk J W, Lee W H, Lee J, Chou H, Piner R D, Hao Y, Akinwande D and Ruoff R S 2013 Enhancement of the electrical properties of graphene grown by chemical vapor deposition via controlling the effects of polymer residue Nano Lett. 13 1462-7

[39] Pirkle A, Chan J, Venugopal A, Hinojos D, Magnuson C, McDonnell S, Colombo L, Vogel E, Ruoff R and Wallace R 2011 The effect of chemical residues on the physical and electrical properties of chemical vapor deposited graphene transferred to $\mathrm{SiO}_{2}$ Appl. Phys. Lett. 99122108

[40] Sze S 1981 Physics of Semiconductor Devices (New York: Wiley)
[41] Zhang W, Hu R, Li D, Huo M-M, Ai X-C and Zhang J-P 2012 Primary dynamics of exciton and charge photogeneration in solvent vapor annealed P3HT/PCBM films J. Phys. Chem. C 116 4298-310

[42] Tamai Y, Ohkita H, Benten H and Ito S 2015 Exciton diffusion in conjugated polymers: from fundamental understanding to improvement in photovoltaic conversion efficiency J. Phys. Chem. Lett. 6 3417-28

[43] Ni Z, Ma L, Du S, Xu Y, Yuan M, Fang H, Wang Z, Xu M, Li D and Yang J 2017 Plasmonic silicon quantum dots enabled high-sensitivity ultrabroadband photodetection of graphene-based hybrid phototransistors ACS Nano 11 9854-62

[44] Guo N, Hu W, Jiang T, Gong F, Luo W, Qiu W, Wang P, Liu L, Wu S and Liao L 2016 High-quality infrared imaging with graphene photodetectors at room temperature Nanoscale 8 16065-72

[45] Miao J, Hu W, Guo N, Lu Z, Liu X, Liao L, Chen P, Jiang T, Wu S and Ho J C 2015 High-responsivity graphene/InAs nanowire heterojunction near-infrared photodetectors with distinct photocurrent on/off ratios Small 11 936-42 